\title{
Nilpotency and related properties of group extensions
}

\author{
David Shield
}

Gilbert Baumslag has given sufficient conditions for a group $G$ to be nilpotent in terms of properties of a normal subgroup $H$ and the quotient $G / H$, and has shown these conditions to be necessary when $G$ is a wreath product and $H$ its base group. His results are extended here to give corresponding conditions for weaker properties ranging from "bounded local nilpotency" to "being an Engel group". Again we ask whether the sufficient conditions found are necessary when $G$ is non-trivially a wreath product, or is of the form $F / \underline{\underline{v}}(R)$; the answer is "yes" for the stronger properties considered, but for the weaker ones the question remains open.

\section{Introduction}

Related to

(i) being nilpotent, but progressively weaker, are the group properties:

(ii) being locally nilpotent,

(iii) having all $k$-generator subgroups nilpotent for some fixed integer $k$ greater than 1 , and

(iv) being an Engel group.

Received 16 September 1970. Communicated by Hanna Neumann. The results reported here form part of the author's M.A. thesis at the Australian National University. The author thanks Professor Hanna Neumann and $\operatorname{Dr}$ M.F. Newman for their encouragement and guidance in the preparation both of the thesis and of this report. 
The class of all groups having any one of these properties is not a variety.

However, corresponding to each, there is a bounded, varietal property. These are:

(i)' being nilpotent of class at most $c$ for a fixed positive integer $c$,

(ii)' having all $k$-generator subgroups nilpotent of class at most $c(k)$ for all $k$, where $c$ is a function on the positive integers; this we will call being boundedly locally nilpotent;

(iii)' having all $k$-generator subgroups nilpotent of class at most $c$ for fixed integers $k$ and $c$, and

(iv)' satisfying an Engel condition.

While each group having the property (i) also has (i)', a group $G$ may have one of the properties (ii), (iii) or (iv) without having the corresponding bounded property. In this case there are groups in the variety generated by $G$ - for example, the countably infinite Cartesian power of $G$ - which do not have the property. In a variety in which every group has one of the properties (i) to (iv), the corresponding bounded property holds uniformly.

This paper investigates conditions for a group $G$ to have each of these properties in terms of properties of a normal subgroup $H$ and the quotient group $G / H$. In general, of course, the only necessary condition is that both $H$ and $G / H$ should have the required property; however for groups non-trivially of the form $F / \underline{\underline{V}}(R)$ (described and investigated in Section 1) and for standard wreath products (in Section 2) a necessary condition much more closely related to the general sufficient condition (of Section 3) is obtained. Baumslag [2] has already obtained a sufficient condition for nilpotency of a general group extension, which he shows is also necessary in the case of the wreath product; that it is necessary in the case of groups of the form $F / \underline{\underline{V}}(R)$ follows from a result of Śmel'kin [10]. We show here that the corresponding conditions for properties ( $i i)$ and ( $i i)$ ' are again both necessary and sufficient. Whether the necessary and the sufficient conditions obtained in the other 
cases are perhaps equivalent remain open questions, which are among those discussed later in Section 5.

It may illuminate the proofs of sections 1 and 2 to point out, in imprecise terms, features shared by the (non-splitting) extensions of the form $F / \underline{V}(R)$ and the (splitting) wreath products, but not by extensions in general. In both cases, all elements outside the normal subgroup have a genuine effect on the normal subgroup, inducing non-trivial automorphisms. In both it is possible to select an element of the normal subgroup (a free generator of $R$ in $F / \underline{V}(R)$; an element of one coordinate subgroup of the wreath product) whose conjugates by distinct (modulo the normal subgroup) powers of an element outside it satisfy two properties; firstly independence, in that no power of any one of them can be expressed as a product of powers of the others; and secondly, commutativity of their images in some homomorphic image of the group in which these images retain the same orders as the originals.

\section{Necessary conditions in $F / \underline{V}(R)$}

THEOREM 1.1. If $P$ is one of the properties (i) to (iv) and $P^{\prime}$ is the corresponding bounded property, $\underline{\underline{\mathrm{V}}}$ is a non-trivial variety, $R$ is a proper normal subgroup of the non-cyclic (absolutely) free group $F$, and $E / \underline{\underline{V}}(R)$ has property $P$, then for some prime $p$, the quotient group $F / R$ is a p-group with property $P$ and $\underline{V}$ is a variety of p-power exponent whose relatively free group $R / \underline{\underline{V}}(R)$ has property $P$ and hence $P^{\prime}$.

If $P$ is the property (i), then moreover $F / R$ is finite.

The last section of the theorem is proved by Śmel'kin [10], Theorem 4.1.

Note that $\underline{\underline{V}}$ itself has property $P^{\prime}$ if $R$ has sufficiently high rank (infinite for (ii)', at least $k$ for (iii)', and at least 2 - which is always the case - for (iv)'. For (i) the rank of $R$ should be strictly greater than the class of $R / \underline{\underline{V}}(R)$, which is bounded above by the class of $F / \underline{\underline{v}}(R))$.

Proof. Each of the properties (i) to (iv) is inherited by both subgroups and homomorphic images. Hence $F / R$ and $R / \underline{\underline{v}}(R)$ both have property $P$. To complete the proof, it suffices to show that both are 
p-groups when $F / \underline{V}(R)$ is an Engel group.

Let $n \in F \backslash R$, and $L=\operatorname{sgp}\{R, \eta\}$. Now $R \unlhd L$ (since $R \subseteq F$ ), $L / R$ is cyclic, and $L$ and $R$ are both free groups. By Lemma 43.42 of [8] we may, if $\eta$ has finite order $t$ modulo $R$, choose a set of generators $\{\alpha, \beta, \ldots\}$ of $L$ such that $\left\{\beta^{t}, \alpha, \alpha^{\beta}, \ldots, \alpha^{\beta^{t-1}}\right\}$ is part of a set of generators of $R$. If $n$ has infinite order modulo $R$, the same procudure gives $\left\{\alpha^{\beta^{i}} \mid i\right.$ an integer $\}$ as part of a set of free generators of $R$. In each case, let $R^{*}$ be the subgroup of $R$ generated by the subset of generators shown, and let $L^{*}=\operatorname{sgp}\left\{R^{*}, \beta\right\}$. It follows from 12.62 of [8] that $\underline{\underline{\mathrm{V}}}\left(R^{*}\right)=\underline{\underline{\mathrm{V}}}(R) \cap R^{*}$. Clearly $L^{*}$ is a free group of rank two generated by $\alpha$ and $\beta$, and $R^{*} \leq L^{*}$.

The next step is to show that the case in which $\eta$, and hence $\beta$, has infinite order modulo $R^{*}$ can not occur. Since $F / \underline{\underline{V}}(R)$ is an Engel group, there must be some positive integer, $l$, such that $[\alpha, Z \beta] \in \underline{\underline{V}}(R)$, and since $\alpha \in R^{*} \leq L^{*}$ and $\beta \in L^{*}$, this means that $[\alpha, Z \beta] \in \underline{\underline{\mathrm{V}}}(R) \cap R^{*}=\underline{\underline{\mathrm{V}}}\left(R^{*}\right) \subseteq(\underline{\underline{\mathrm{V}}} \cap \underline{\underline{\mathrm{A}}})\left(R^{*}\right)$. However since $R^{*} /(\underline{\underline{\mathrm{V}}} \cap \underline{\underline{\mathrm{A}}})\left(R^{*}\right)$ is abelian, a simple induction on $l$ shows that modulo $(\underline{\underline{V}} \cap \underline{\underline{A}})\left(R^{*}\right)$,

$$
[\alpha, Z \beta] \equiv \alpha^{(\beta-1)^{2}}=\prod_{0 \leq i \leq Z} \alpha^{n(i) \beta^{i}}
$$

where $n(i)=(-1)^{2-i}\left(\begin{array}{l}2 \\ i\end{array}\right)$. If $\beta$ has infinite order modulo $R^{*}$, the conjugates of $\alpha$ whose powers occur in this product are distinct elements of a set of free generators of $R^{*}$, and one of the powers (corresponding to $i=0$ ) is equal to $\alpha^{ \pm 1}$ and is therefore non-trivial modulo the verbal subgroup $(\underline{\underline{V}} \cap \underline{\underline{A}})\left(R^{*}\right)$. Hence the Engel commutator $[\alpha, Z \beta]$ is

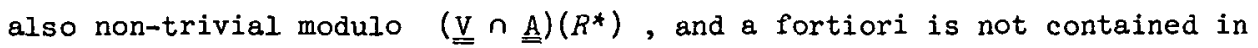
$\underline{\underline{V}}\left(R^{*}\right)$.

This contradiction shows that $F / R$ is periodic, and $B$ has finite order, $t$ say, modulo $R$ and also modulo $R^{*}$. The theorem will be proved if we show that $t$ and the exponent of $\underline{\underline{V}}$ are powers of the same prime.

If they are not, there exist distinct primes $p$ and $q$ such that 
$t=u q$ for some integer, $u$, and $\stackrel{\mathrm{A}}{p} \subseteq \underline{\mathrm{V}}$. Now $\stackrel{\mathrm{A}}{\Rightarrow}\left(R^{*}\right) \supseteq \underline{\underline{V}}\left(R^{*}\right)$, and as before, for some positive integer, $l$, the Engel commutator $[\alpha, Z B]$ in the free generators of $L^{*}$ is contained in $\underline{\underline{V}}(R) \cap R^{*}$ and so in $\underset{\Rightarrow p}{A}\left(R^{*}\right)$. Hence $L^{*} / \mathrm{A}_{p}\left(R^{*}\right)$ is an Engel group; but it is finite, of order $t p^{t+1}$, and so by the well-known result of Zorn and Zassenhaus (reported without proof in [11]) is nilpotent. It can easily be checked that $\alpha$ and $\beta^{\text {up }}$ are, modulo $\mathrm{A}_{p}\left(R^{*}\right)$, elements of orders $p$ and $q$ respectively which do not commute (since $\alpha$ and $\alpha^{\beta^{2 \varphi}}$ are distinct free generators of $R^{*}$ ). But this contradicts the nilpotency of $L^{*} / \stackrel{A}{=p}\left(R^{*}\right)$.

COROLLARY 1.2. If $\mathrm{P}^{\prime}$ is one of the properties (i)' to (iv)', $\underline{\underline{V}}$ is a non-trivial variety, $R$ is a proper normal subgroup of the non-cyclic (absolutely) free group $F$, and $F / \underline{\underline{V}}(R)$ has property $P^{\prime}$, then for some prime $p$ both $F / R$ and the relatively free group $R / \underline{\underline{\mathrm{V}}}(R)$ have $p$-power exponent and the property $P^{\prime}$.

Proof. As before, $F / R$ and $R / \underline{\underline{V}}(R)$ inherit the property $P^{\prime}$. All other consequences except a bound on the order of elements of $F / R$ follow immediately from Theorem 1.1. If now $l$ is the Engel length or the bound on the class of two-generator subgroups, so that for arbitrary $\alpha, \beta \in F$ we have $[\alpha, Z \beta] \in \underline{\mathrm{y}}(R)$, then $l$ is the required bound. For if $\alpha \in R$, and $\beta$ has order $t$ modulo $R$, then modulo $(\underline{\underline{V}} \cap \underline{\underline{A}})(R)$ we have

$$
[\alpha, Z \beta] \equiv \prod_{0 \leq i \leq l} \alpha^{n(i) \beta^{i}}
$$

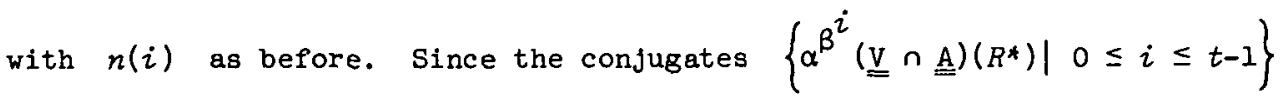
are distinct, the product can be trivial modulo $(\underline{\underline{V}} \cap \underline{\underline{A}})\left(R^{*}\right)$ only if $t-1<2$, whence $t \leq 2$, as claimed.

\section{Necessary conditions in A wr B}

Similar results hold for the wreath product. That relating to nilpotency is part of a well-known result of Gilbert Baumslag; those for bounded and unbounded Engel properties and for local nilpotency are due to 
R.B.J.T. Allenby [1]. (I owe this reference to James wiegold.)

THEOREM 2. [Baumslag [2], Allenby [1].] If $P$ is one of the properties (i) to (iv), $A$ and $B$ are non-trivial groups, and $A$ wr $B$ has property $P$, then for some prime $p$, both $A$ and $B$ are $p$-groups with property $P$.

If $P^{\prime}$ is one of the properties (i)' to (iv)', $A$ and $B$ are non-trivial groups, and $A$ wr $B$ has property $P^{\prime}$, then for some prime $p$, both groups $A$ and $B$ are of $p$-power exponent with property $P^{\prime}$.

If in addition $P$ (or $P^{\prime}$ ) stands for nilpotency, then $B$ is a finite group.

Proof. The proofs are at first similar to those for Theorem 1.1 and Corollary 1.2. This time $\alpha$ is chosen as an arbitrary non-trivial element of the first coordinate subgroup $A(1)$ of the base group of $A$ wr $B$, and $B$ as an arbitrary non-trivial element of the top group. The argument takes place in the subgroup of $A$ wr $B$ generated by $\alpha$ and B.

Two additional sections of proof are required. In the second part of the theorem, if $l$ is the Engel length or the bound on the class of two-generator subgroups of $A$ wr $B$, then in the expression

$$
[\alpha, z \beta]=\prod_{0 \leq i \leq Z} \alpha^{n(i) \beta^{i}}
$$

the power to which each distinct conjugate $\alpha^{\beta^{i}}$ for $0 \leq i<|\beta|$ is raised is a sum of binomial coefficients $\left(\begin{array}{l}l \\ i\end{array}\right)$ which cannot exceed $2^{2-1}$; this value is therefore a bound on the exponent of the group $A$. For the third part of the theorem, the finiteness of $B$ was proved by Baumslag [2], Corollary 3.2.

\section{Sufficient conditions}

We turn now to arbitrary extensions. The case of nilpotency is familiar :

THEOREM 3.1. [Baumslag, [2], Lemma 3.8.] An extension of a 
nilpotent group of $p$-power exponent by a finite p-group is nilpotent.

A new upper bound on the class of such extensions will be given in a subsequent paper which is being prepared for publication. This bound is always attained by the wreath product, and provides a solution of the problem of the exact class of nilpotent wreath products. Upper and lower bounds for the class of nilpotent wreath products have been obtained earlier by Liebeck [4], Meldrum [5], Scruton [9], Morley [7], and Meldrum [6].

THEOREM 3.2. If $P$ is one of the properties (ii) to (iv) and $p$ is a prime, then an extension of a locally nilpotent p-group by a p-group with property $P$ has property $P$.

Proof. Let $H$ be a locally nilpotent $p$-group which is a normal subgroup of $G$, such that $G / H$ is a $p$-group with property $P$. If $P$ is (ii), let $G^{*}$ be any finitely-generated subgroup of $G$; if $P$ is (iii) let $G^{*}$ be a k-generator subgroup of $G$. In either case, $G^{*} / H \cap G^{*} \cong G^{*} H / H \leq G / H$ is nilpotent and finite. Then $H \cap G^{*}$ is a subgroup of $H$ with finite index in the finitely generated group $G^{*}$, so is also finitely generated, therefore nilpotent, and hence finite. This means that $G^{*}$ is a finite $p$-group and is therefore nilpotent, and the results for properties (ii) and (iii) follow.

If $P$ is (iv), let $\alpha, \beta$ be arbitrary elements of $G$. Since $G / B$ is an Engel group, there is a positive integer $\tau_{0}$ such that $\gamma=\left[\alpha, Z_{0} \beta\right] \in H$. Let $L=\operatorname{sgp}\{\gamma, \beta\}$ and let $H^{*}$ be the normal closure of $\gamma$ in $L$. Since $H \cap L \triangleleft L$, it follows that $H^{*} \leq H \cap L$, and so $H^{*}$ is a locally nilpotent $p$-group. The element $B$ has finite $p$-power order, $q$ say, modulo $H^{*}$, and $H^{*}$ is generated by the finite set $\left\{\beta^{q}\right\} \cup\left\{\gamma^{\beta^{i}} \mid 0 \leq i \leq q-1\right\}$; and so $H^{*}$ is nilpotent, and therefore finite. Now $L$, being an extension of $H^{*}$ by a $q$-cycle, is also a finite $p$-group, and therefore nilpotent. Hence $\left[\gamma, Z_{1} \beta\right]=1$ for some positive integer $l_{1}$, and $\left[\alpha, l_{B}\right]=1$ where $l=l_{0}+l_{1}$. Thus $G$ is in this case an Engel group, as claimed.

COROLLARY 3.3. If $P^{\prime}$ is one of the properties (ii)' to (iv)' and $p$ is a prime, then an extension of a boundedly locally nilpotent grow of 
p-power exponent by a group of p-power exponent with property $P^{\prime}$ has property $P^{\prime}$.

Proof. Let $H$ be a boundedly locally nilpotent group of $p$-power exponent which is normal in $G$, such that $G / H$ has $p$-power" exponent and property $P^{\prime}$. Let $\underline{\underline{\mathrm{V}}}$ be the variety generated by $H$ and $\underline{\underline{W}}$ the variety generated by $G / H$; then $\underline{\underline{V}}$ and $\underline{W}$ have properties (ii)' and $P^{\prime}$ respectively, and both have $p$-power exponent. From Theorem 3.2, every group in the product variety $\underline{\underline{V W}}$ has the unbounded property corresponding to $P^{\prime}$, and hence, by the remarks made in the introduction, every group in $\underline{\underline{V W}}$, and in particular $G$, has property $P^{\prime}$.

\section{Applications to product varieties}

THEOREM 4. If $P^{\prime}$ is one of the properties (ii)' to (iv)', then the product $\underline{\underline{V W}}$ of the non-trivial varieties $\underline{\underline{V}}$ and $\mathrm{W}$ has property $\mathrm{P}^{\prime}$ if $\underline{\underline{V}}$ has property (ii)', W has property $\mathrm{P}^{\prime}$, and both have exponents equal to powers of one prime, $p$; and $\underline{\underline{V W}}$ has property $P^{\prime}$ only if $\underline{\underline{V}}$ and W both have property $\mathrm{Pl}^{\prime}$ and both have p-power exponent.

It is well-known that the product of non-trivial varieties can never be nilpotent; see for example Theorem 24.23 of [8] and Corollary 4.2 of [10].

Proof. Since $F / \underline{\underline{V}}(\underline{\underline{W}}(F))$ is relatively free with the same rank as $F$ in the variety $\underline{\underline{V W}}$ (for example by 21.12 of [8]), the sufficient condition follows fram Corollary 3.3. The necessary condition follows from Corollary 1.2 , but is more easily proved by noting that if $\underline{\underline{V}}$ and $\underline{\underline{W}}$ do not both have $p$-power exponent, there exist distinct primes $q$ and $q^{\prime}$ such that $c_{q} \in \underline{\underline{\mathrm{V}}}, C_{q^{\prime}} \in \underline{\underline{\mathrm{W}}}$, and hence the non-Engel group $C_{q} \mathrm{wr} C_{q^{\prime}}$ belongs to $\underline{\underline{\mathrm{VW}}}$.

\section{Problems}

This paper raises, and leaves unanswered, several interesting questions about the gaps between corresponding necessary and sufficient conditions.

Firstly, is it true that every Engel variety of prime-power exponent is locally nilpotent? This is a restriction of the well-known unsolved 
problem whether every Engel variety is locally nilpotent.

Secondly, a closely related question: is a variety necessarily locally nilpotent if all $k$-generator groups are nilpotent of class $c$ ? Golod in [3] and M.F. Newman (unpublished) have independently constructed two-generator infinite Engel $p$-groups, and $(k+1)$-generator $p$-groups which are not nilpotent but all of whose $k$-generator subgroups are nilpotent. However, none of these have finite exponent; and there is no bound on the Engel length in the first case, or on the class of the nilpotent $k$-generator subgroups in the second. Affirmative answers to the first two questions would mean that the necessary conditions of Theorem 1.1, Corollary 1.2, Theorem 4, and of the second part of Theorem 2 are all sufficient.

Thirdly, to cover the first part of Theorem 2: if $H$ is an Engel p-group and $G / H$ is (to take the simplest case) a p-cycle, does it follow that $G$ is an Engel group? Even more restricted forms of this question, where $H$ is made to satisfy an Engel condition or to have prime-power exponent, appear very difficult; an affirmative answer to the first question above would solve the problem in the case where both extra conditions are imposed.

Finally, does there exist an Engel group of finite (prime-power) exponent which does not satisfy an Engel condition? This question is raised by the observation that every extension, $G$ say, of a boundedly locally nilpotent $p$-group $H$ by a boundedly Engel $p$-group $G / H$ is boundedly Engel unless it fails to have finite exponent; and among the examples of unboundedly Engel groups known to me, not one has finite exponent.

\section{References}

[1] R.B.J.T. Allenby, "Adjunction of roots to groups", (M.Sc.Tech. thesis, University of Manchester, 1963).

[2] Gilbert Baumslag, "Wreath products and p-groups", Proc. Combridge Phizos. Soc. 55 (1959), 224-231. 
[3] E.S. Golod, "Nil-algebras and residually finite p-groups" (Russian), Izv. Akad. Nauk SSSR Ser. Mat. 28 (1964), 273-276.

[4] Hans Liebeck, "Concerning nilpotent wreath products", Proc. Combridge Philos. Soc. 58 (1962), 443-451.

[5] J.D.P. Meldrum, "Central series in wreath products", Proc. Cambridge Phizos. Soc. 63 (1967), 551-567.

[6] J.D.P. Meldrum, "On nilpotent wreath products", Proc. Combridge Philos. Soc. 68 (1970), 1-15.

[7] Larry Morley, "Bounds on the nilpotency class of certain semi-direct products", submitted to Trans. Amer. Math. Soc.

[8] Hanna Neumann, Varieties of groups (Ergebnisse der Mathematik und ihrer Grenzgebiete, Band 37, Springer-Verlag, Berlin, Heidelberg, New York, 1967).

[9] Teresa Scruton, "Bounds on the class of nilpotent wreath products", Proc. Combridge Philos. Soc. 62 (1966), 165-169.

[10] A.L. Śmel'kin, "Wreath products and varieties of groups" (Russian), Izv. Akad. Nauk SSSR Ser. Mat. 29 (1965), 149-170.

[11] Max Zorn, "Nilpotency of finite groups", Buzl. Amer. Math. Soc. 42 (1936), 485.

School of General Studies,

Australian National University,

Canberra, ACT. 